\title{
Stress MPI, coronary CTA, and multimodality for subsequent risk analysis
}

\author{
Masanao Naya, MD, PhD, a and Nagara Tamaki, MD, PhD \\ ${ }^{a}$ Hokkaido University Graduate School of Medicine, Sapporo, Japan
}

Received Dec 23, 2015; accepted Dec 23, 2015

doi: $10.1007 / \mathrm{s} 12350-016-0400-z$

\section{See related article, pp. 185-197}

The initial diagnosis and assessment of coronary artery disease (CAD) are important in risk stratification for future cardiovascular events and subsequent treatment choice. The current diagnostic approach to CAD is mainly based on anatomic and functional imaging. Stress myocardial perfusion imaging (MPI) or/and coronary CT angiography (CCTA) are commonly used for symptomatic chronic CAD patients. MPI is useful for detecting ischemia and assessing the severity of CAD. ${ }^{1}$ MPI is generally used for determining the appropriate approach to coronary intervention. In addition, semiquantitative analysis of myocardial ischemia by MPI provides highly useful information regarding the future outcomes. ${ }^{2,3}$ CCTA, on the other hand, is rapidly becoming a commonly used technique for diagnosing CAD with high accuracy. ${ }^{4,5}$ In particular, CT has been reported to have a high negative predictive value for suspected CAD and a high prognostic value. ${ }^{6-8}$ Therefore, CCTA is being increasingly used as an alternative to MPI for the initial diagnosis of CAD, particularly in patients with low to intermediate probability of CAD.

In several recent studies, MPI and CCTA for risk analysis of stable CAD were compared. ${ }^{9-11}$ The data obtained indicate comparative results in terms of longterm outcomes. However, a higher rate of revascularization is noted for patients who underwent CCTA than those who underwent MPI. These findings suggest that both noninvasive tests for detecting and excluding CAD are comparable in terms of diagnostic accuracy. MPI

Reprint requests: Nagara Tamaki MD, PhD, Hokkaido University Graduate School of Medicine, Sapporo, Japan; natamaki@med. hokudai.ac.jp

J Nucl Cardiol 2016;23:198-201.

$1071-3581 / \$ 34.00$

Copyright (C) 2016 American Society of Nuclear Cardiology. and CT provide information regarding different aspects of the disease-atherosclerotic and ischemic changes, respectively; thus, both tests complement each other. The higher rate of revascularization in CCTA may be explained by the tendency of cardiologists to consider coronary intervention when the CT performed initially shows significant coronary stenosis, whereas such an intervention may not be considered when the MPI performed initially does not show ischemia. Low-risk patients enrolled in our observational J-COMPASS Study in Japan also showed such a tendency. ${ }^{11}$ The incidences of MACE including death, acute myocardial infarction, cardiac event, and late revascularization were $7.0 \%, 2.6 \%$, and $2.1 \% /$ year when catheter, MPI, and CCTA were initially performed, respectively. The percentages of patients who underwent catheter after the initial tests by MPI and CCTA were $33 \%$ and $31 \%$, respectively. The hazard ratio for early revascularization in CCTA compared with that in MPI was 1.62 (95\% confidence interval: 1.20-2.08) and that for catheter was 5.36 (95\% CI: 4.07-7.05).

In this issue of the Journal of Nuclear Cardiology, Cantoni et al. report on the first meta-analysis they conducted to compare the long-term prognostic values of MPI and CCTA for adverse cardiovascular events in patients with suspected or known CAD (XXX). Their meta-analysis confirmed similar long-term prognostic values of MPI and CCTA. In the MPI group, cardiac hard events occurred at $1.1 \%$ /year in patients with normal MPI findings and 2.9\%/year in those with abnormal MPI findings [pooled hazard ratio, 2.32 (95\% confidence interval, 1.952.75)]. In the CCTA group, cardiac hard events occurred at $0.7 \%$ year in patients with nonsignificant stenosis and $4.6 \% / y e a r$ in those with significant coronary stenosis of more than $50 \%$ [pooled hazard ratio, 4.35 (95\% confidence interval, 2.66-7.13), $P=$ NS compared with MPI]. On the other hand, the hazard ratio for the occurrence of a combination of endpoints including late revascularization was higher in the CCTA group than in the MPI group. In the MPI group, combined events occurred in $1.3 \%$ year of 
A

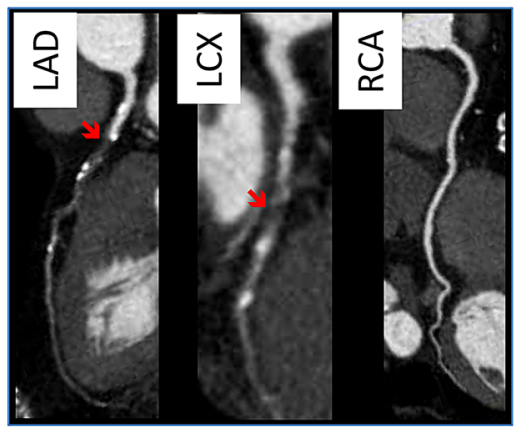

B

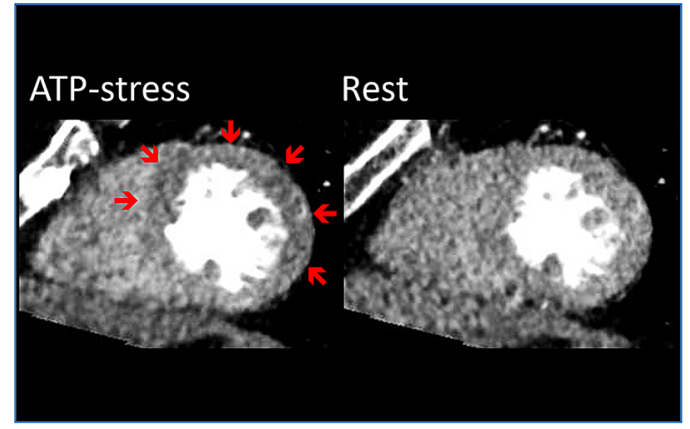

C
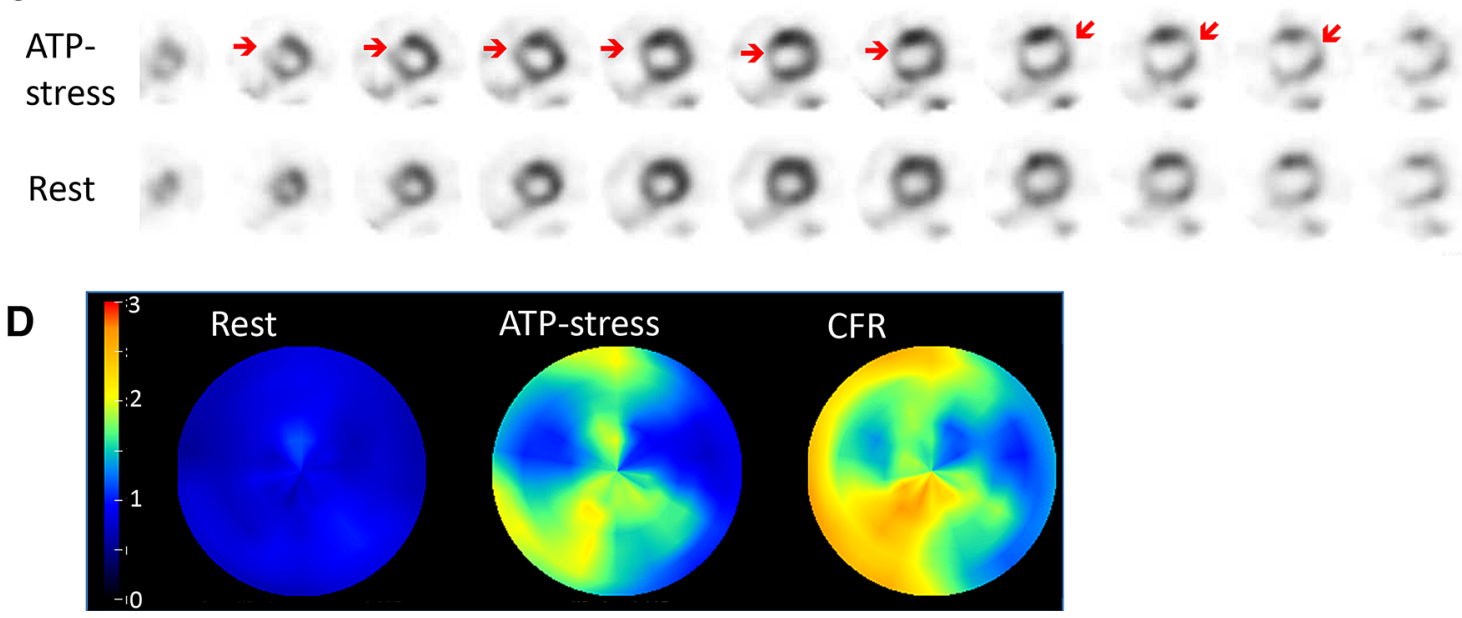

Figure 1. Imaging results for a male patient (approximately 70 years old) was referred for evaluation of typical chest pain. Selected CT curved multiplanar views of left and right coronary arteries (A). Images show extensive and severe CAD involving the proximal left anterior descending (100\% stenosis) and mid left circumflex coronary (LCx) arteries (100\% stenosis). Stress CT perfusion imaging during adenosine triphosphate (ATP) infusion shows ischemia in the area corresponding to the coronary artery stenosis $(\mathbf{B})$. Short-axis stress and rest myocardial perfusion SPECT images show transit ischemic dilation of left ventricle and severe perfusion defect involving anterior-lateral left ventricular wall but was mostly reversible (C). Patient's quantitative global rest and stress myocardial blood flow, and CFR determined by 015-water PET were 0.84 and $1.45 \mathrm{~mL} / \mathrm{g} / \mathrm{min}$, and 1.73, respectively (D), and the coronary calcium score was 661. CT angiography and CT perfusion comprehensively show the anatomical and functional disease burden for assessing the severity of CAD. PET/CT provides quantitative CFR and calcium score, which might improve risk stratification.

patients with normal MPI findings and 5.2\%/year of abnormal MPI findings [pooled hazard ratio, 2.71 (95\% CI, 1.52-4.83)]. However, in the CCTA group, the event rate increased to $7.9 \% / y$ ear in patients with obstructive disease compared with $0.8 \%$ year in those with nonobstructive disease [pooled hazard ratio, 7.61 (95\% CI, 3.5116.53], $P<0.05$ compared with MPI]. Although the authors did not provide possible reasons for these outcomes in their discussion, the main reason may have been explained by previous reports. Although the final outcome may not be significantly different between the two methods so far, we should carefully analyze, given the higher frequency of revascularization in the CCTA group, whether CCTA might be more or less beneficial for CAD patients. In this respect, long-term outcome studies may be required.

Although both the MPI and CCTA tests are effective for excluding CAD in patients with low and intermediate pretest probabilities in terms of long-term outcomes, MPI may be better for patients in whom contrast medium should be used with great caution and for those who are considered to have nondiagnostic findings such as high levels of coronary artery calcification prior to any CT examinations. Tests with lower radiation dosages could be another reason for selecting the imaging modality. Recently, 256-320 multislice CT with reconstruction using iterative approximation and DSPECT realize low radiation dosages. 
On the other hand, the evaluation of the severity of CAD (i.e., more than $10 \%$ ischemia on MPI or twovessel disease on CCTA) is another issue in addition to the diagnosis of CAD in relation to the selection of appropriate treatments such as optimal medical therapy, percutaneous coronary intervention (PCI), and coronary artery bypass grafting $(\mathrm{CABG})$. Indeed, in patients with multivessel $\mathrm{CAD}$ and diabetes mellitus, $\mathrm{CABG}$ is superior to PCI for improving prognosis. ${ }^{12,13}$

CCTA and MPI are complementary in assessing $\mathrm{CAD}$ burden once severe $\mathrm{CAD}$ is detected. For example, when more than $10 \%$ ischemia is observed by MPI, the location and number of stenotic sites assessed by CCTA or invasive catheter are the next concerns for cardiologists. Significant coronary stenosis in the proximal LAD or left main trunk may predict worse prognosis compared with the stenosis in branch arteries if the severity of ischemia is similar. On the other hand, when intermediate stenosis in the proximal LAD or left main trunk is observed by CCTA, the assessment of functional ischemia by stress MPI will be the next step. These different aspects of imaging tests suggest that in cases of moderate to severe, but not mild CAD, both the anatomic burden on $\mathrm{CT}$ and the ischemic burden on stress MPI could be often desirable. Recent CT examination enables us to simultaneously evaluate ischemia and coronary anatomy by stress perfusion CT and CTA. ${ }^{14,15}$ Moreover, fractional flow reserve estimation by CT (FFR_CT) for functional analysis is receiving much attention. FFR_CT increases the specificity for identifying positivity for invasive FFR compared with CCTA alone. ${ }^{16,17}$ Likewise, MRI provides stress MPI, scar imaging, and coronary angiography. ${ }^{18,19}$ Accordingly, cardiologists are interested in the simultaneous assessment of anatomical information and functional ischemia owing the latest technologies (Figure 1). PET/CT and SPECT/CT also provide information on myocardial ischemia and coronary artery calcium burden. Furthermore, PET/CT is the established method to assess quantitative myocardial blood flow and coronary flow reserve, which can greatly improve risk stratification of CAD. ${ }^{20-22}$

From the above, both CTA and MPI as the initial tests are comparable in identifying and excluding the presence of CAD. However, in patients highly likely having or known to have CAD, information on both anatomic and ischemic burden could be useful for considering the strategy to improve prognosis. On the other hand, soft plaques with positive morphologic remodeling shown by $\mathrm{CCTA}^{23}$ and $\mathrm{MRI}^{24}$ are also considered for identifying patients at high risk CAD, but the appropriate use criteria of plaque assessment is as yet not established in the clinical settings.
Although MPI or CCTA alone, or both should be used appropriately considering the balance between diagnostic value, prognostic value, medical cost, and patients' burden, it would be valuable to realize complementary assessment of anatomy and functional ischemia by using the latest technologies in patients with $\mathrm{CAD}$ in the near future.

\section{Disclosure}

The authors have no financial conflict of interest.

\section{Reference}

1. Hendel RC, Berman DS, Di Carli MF, Heidenreich PA, Henkin RE, Pellikka PA, et al. ACCF/ASNC/ACR/AHA/ASE/SCCT/ SCMR/SNM 2009 Appropriate Use Criteria for Cardiac Radionuclide Imaging: A Report of the American College of Cardiology Foundation Appropriate Use Criteria Task Force, the American Society of Nuclear Cardiology, the American College of Radiology, the American Heart Association, the American Society of Echocardiography, the Society of Cardiovascular Computed Tomography, the Society for Cardiovascular Magnetic Resonance, and the Society of Nuclear Medicine. J Am Coll Cardiol 2009;53:2201-29.

2. Elhendy A, Schinkel AF, van Domburg RT, Bax JJ, Valkema R, Huurman A, et al. Risk stratification of patients with angina pectoris by stress $99 \mathrm{mTc}$-tetrofosmin myocardial perfusion imaging. J Nucl Med 2005;46:2003-8.

3. Shaw LJ, Iskandrian AE. Prognostic value of gated myocardial perfusion SPECT. J Nucl Cardiol 2004;11:171-85.

4. Budoff MJ, Dowe D, Jollis JG, Gitter M, Sutherland J, Halamert $\mathrm{E}$, et al. Diagnostic performance of 64-multidetector row coronary computed tomographic angiography for evaluation of coronary artery stenosis in individuals without known coronary artery disease: results from the prospective multicenter ACCURACY (Assessment by Coronary Computed Tomographic Angiography of Individuals Undergoing Invasive Coronary Angiography) trial. J Am Coll Cardiol 2008;52:1724-32.

5. Abdulla J, Abildstrom SZ, Gotzsche O, Christensen E, Kober L, Torp-Pedersen C. 64-multislice detector computed tomography coronary angiography as potential alternative to conventional coronary angiography: a systematic review and meta-analysis. Eur Heart J 2007;28:3042-50.

6. Abdulla J, Asferg C, Kofoed KF. Prognostic value of absence or presence of coronary artery disease determined by 64-slice computed tomography coronary angiography a systematic review and meta-analysis. Int J Cardiovasc Imaging 2011;27:413-20.

7. Hulten EA, Carbonaro S, Petrillo SP, Mitchell JD, Villines TC. Prognostic value of cardiac computed tomography angiography: A systematic review and meta-analysis. J Am Coll Cardiol 2011;57:1237-47.

8. Min JK, Shaw LJ, Devereux RB, Okin PM, Weinsaft JW, Russo DJ, et al. Prognostic value of multidetector coronary computed tomographic angiography for prediction of all-cause mortality. J Am Coll Cardiol 2007;50:1161-70.

9. Douglas PS, Hoffmann U, Patel MR, Mark DB, Al-Khalidi HR, Cavanaugh $\mathrm{B}$, et al. Outcomes of anatomical versus functional 
testing for coronary artery disease. New Engl J Med 2015;372: 1291-300.

10. Hlatky MA, Shilane D, Hachamovitch R, Dicarli MF, Investigators S. Economic outcomes in the Study of Myocardial Perfusion and Coronary Anatomy Imaging Roles in Coronary Artery Disease registry: The SPARC Study. J Am Coll Cardiol 2014;63:1002-8.

11. Yamauchi $\mathrm{T}$, Tamaki N, Kasanuki H, Kimura T, Uemura $\mathrm{Y}$, Iimuro S, et al. Optimal initial diagnostic strategies for the evaluation of stable angina patients: A multicenter, prospective study on myocardial perfusion imaging, computed tomographic angiography, and coronary angiography. Circ J 2012;76:2832-9.

12. Farkouh ME, Domanski M, Sleeper LA, Siami FS, Dangas G, Mack M, et al. Strategies for multivessel revascularization in patients with diabetes. New Engl J Med 2012;367:2375-84.

13. Serruys PW, Morice MC, Kappetein AP, Colombo A, Holmes DR, Mack MJ, et al. Percutaneous coronary intervention versus coronary-artery bypass grafting for severe coronary artery disease. New Engl J Med 2009;360:961-72.

14. Blankstein R, Shturman LD, Rogers IS, Rocha-Filho JA, Okada DR, Sarwar A, et al. Adenosine-induced stress myocardial perfusion imaging using dual-source cardiac computed tomography. J Am Coll Cardiol 2009;54:1072-84.

15. Kikuchi Y, Oyama-Manabe N, Naya M, Manabe O, Tomiyama Y, Sasaki T, et al. Quantification of myocardial blood flow using dynamic 320-row multi-detector CT as compared with $\mathrm{O} 15-\mathrm{H}_{2} \mathrm{O}$ PET. Eur Radiol 2014;24:1547-56.

16. Koo BK, Erglis A, Doh JH, Daniels DV, Jegere S, Kim HS, et al. Diagnosis of ischemia-causing coronary stenoses by noninvasive fractional flow reserve computed from coronary computed tomographic angiograms. Results from the prospective multicenter DISCOVER-FLOW (Diagnosis of Ischemia-Causing Stenoses Obtained Via Noninvasive Fractional Flow Reserve) study. J Am Coll Cardiol 2011;58:1989-97.
17. Norgaard BL, Leipsic J, Gaur S, Seneviratne S, Ko BS, Ito H, et al. Diagnostic performance of noninvasive fractional flow reserve derived from coronary computed tomography angiography in suspected coronary artery disease: The NXT trial (Analysis of Coronary Blood Flow Using CT Angiography: Next Steps). J Am Coll Cardiol 2014;63:1145-55.

18. Kato S, Kitagawa K, Ishida N, Ishida M, Nagata M, Ichikawa Y, et al. Assessment of coronary artery disease using magnetic resonance coronary angiography: A national multicenter trial. J Am Coll Cardiol 2010;56:983-91.

19. Greenwood JP, Maredia N, Younger JF, Brown JM, Nixon J, Everett CC, et al. Cardiovascular magnetic resonance and singlephoton emission computed tomography for diagnosis of coronary heart disease (CE-MARC): A prospective trial. Lancet 2012;379:453-60.

20. Murthy VL, Naya M, Foster CR, Hainer J, Gaber M, Di Carli G, et al. Improved cardiac risk assessment with noninvasive measures of coronary flow reserve. Circulation 2011;124:2215-24.

21. Naya M, Murthy VL, Foster CR, Gaber M, Klein J, Hainer J, et al. Prognostic interplay of coronary artery calcification and underlying vascular dysfunction in patients with suspected coronary artery disease. J Am Coll Cardiol 2013;61:2098-106.

22. Naya M, Tamaki N, Tsutsui H. Coronary flow reserve estimated by positron emission tomography to diagnose significant coronary artery disease and predict cardiac events. Circ J 2015;79:15-23.

23. Motoyama S, Sarai M, Harigaya H, Anno H, Inoue K, Hara T, et al. Computed tomographic angiography characteristics of atherosclerotic plaques subsequently resulting in acute coronary syndrome. J Am Coll Cardiol 2009;54:49-57.

24. Noguchi T, Yamada N, Higashi M, Goto Y, Naito H. High-intensity signals in carotid plaques on T1-weighted magnetic resonance imaging predict coronary events in patients with coronary artery disease. J Am Coll Cardiol 2011;58:416-22. 